\title{
Factor de forma para la Tectona grandis L.F, empresa MLR-Forestal, Siuna, Costa Caribe Norte de Nicaragua
}

Cesar Augusto Aguilar Rodríguez ${ }^{1}$
Ariel Francisco Sequeira Guillen $^{2}$
Efraín de Jesús Peralta Tercero $^{3}$

\section{Resumen}

Esta investigación se realizó en la empresa MLR-Forestal de Nicaragua, en la comunidad Waspado, municipio de Siuna, Región Autónoma Costa Caribe Norte. Fue un estudio predictivo, transversal y prospectivo, orientado en la determinación del factor de forma de la Tectona grandis L.F.

La muestra equivale a 255 árboles distribuido en 51 parcelas circulares de 1000 $\mathrm{m}^{2}$ en tres fincas (Waspado con un total de 86.28 ha, Danli con 58.87 y Mutiwas con 52.85) para un total de 198 ha, dentro de cada parcela fueron seleccionados 5 árboles, entre dominantes, codominantes y suprimidos. Se obtuvieron los factores de forma por finca de 0.54 para Waspado, 0.57 para Mutiwas y 0.53 para Danli y un factor de forma promedio general para las tres fincas de o.55. El cual difiere del factor de forma nacional para las latifoliadas 0.70 .

Los datos permitieron construir una tabla para estimaciones de volúmenes en plantaciones de TECA. Al encontrar el factor de forma real de la Tectona grandis L.F obtuvimos las siguientes diferencias con relación al porcentaje de error según el factor de forma estipulado por el Instituto Nacional Forestal (INAFOR) y con el modelo matemático encontrado (porcentaje de error según el factor de forma encontrado $(-5.84 \%)$, el estipulado por el INAFOR es de $(19.83 \%)$ y modelo matemático (-11.30 $\%)$, los valores negativos hacen una subestimación de volumen real y los positivos hacen una sobreestimación de volumen.

Palabras clave: Factor de forma; plantaciones forestales; modelos matemáticos, Tectona grandis L.F, estimación de volúmenes.

\footnotetext{
Ingeniero Agroforestal. Correo: aguilarrodriguezc@yahoo.com

Ingeniero Agroforestal. Correo: presidentfes@yahoo.com

Master en Docencia Universitaria. Profesor de la Universidad de las Regiones Autónomas de la Costa Caribe Nicaragüense Recinto Universitario Las Minas. Correo: eperaltat11@hotmail.com.
}

Recibido: 07/02/2017 Aprobado: 23/11/2017 


\section{Summary}

This research was carried out at MLR-Forestal Company of Nicaragua, Waspado Community, Siuna municipality, Autonomous Regions of the North Caribbean Coast. It was a predictive, transversal and prospective study, oriented in the determination of the form factor of Tectona grandis L.F.

The sample is equivalent to 255 trees distributed in 51 circular plots of $1000 \mathrm{~m}^{2}$ in three farms (Waspado with a total of 86.28 ha, Danli with 58.87 and Mutiwas with 52.85) for a total of $198 \mathrm{ha}$, within each plot, 5 trees were selected between dominant, codominant and suppressed trees. For Waspado, 0.54, 0.57 for Mutiwas and 0.53 for Danli and a general average form factor for the three farms of 0.55 form factors that were obtained from each farm. This differs from the national form factor for broadleaf 0.70 .

The data allowed the construction of a table for volume that are estimated in TECA plantations. When finding the real form factor of the Tectona grandis LF we obtained the following differences in relation to the error percentage according to the form factor stipulated by the National Forestry Institute (INAFOR) and the mathematical model found (percentage of error according to the factor of found form $-5.84 \%$ ), stipulated by INAFOR is (19.83\%) and mathematical model (-11.30\%), negative values make an underestimation of real volume and positive values make an overestimation of volume.

Key Words: Form factor; Forest plantations; mathematical models, Tectona grandis L.F, estimation of volumes.

\section{Introducción}

En la actualidad la producción de madera proveniente de plantaciones forestales constituye una actividad de gran importancia, ya que genera ingresos económicos a las familias, se abastece la demanda de madera en el mercado evitando que se continúe con el aprovechamiento de los bosques naturales. La MLR-Forestal ha establecido plantaciones forestales comerciales con la especie Tectona grandis L.F, produciendo beneficios socio-económicos para las zonas rurales donde se desarrollan las actividades, esto como producto de un manejo sustentable y responsable del recurso forestal para lo cual se necesita un factor de forma o una tabla volumétrica para la cubicación de los árboles.

Normalmente cada especie debería de tener su factor de forma para la estimación de volumen para así poder minimizar el margen de error, por lo cual en otras investigaciones anteriores se han calculado factores de forma en Gmelina arbórea donde el factor 
de forma encontrado es de 0.407 el cual presenta valores similares a los puestos por el autor Martin Cuadro Hidalgo que presenta un factor de 0.46 (Castedo, 2011, pág. 47)

Según David A. G. (2013) el factor de forma para la especie teca es 0.55 y para melina es de 0.49. En la actualidad se usa un factor de forma estándar para las especies latifoliadas establecido por el Instituto Nacional Forestal (INAFOR) el cual es de 0.70; La empresa MLR-Forestal posee un componente forestal amplio por tal motivo la determinación del factor de forma para la especie Tectona grandis L.F, es un elemento indispensable y necesario para estimar volúmenes reales y ajustar las expectativas de ingresos futuros a partir de estas plantaciones, por lo que se justifica el presente tema de tesis.

¿Cuál es el factor de forma real de la Teca en las plantaciones de la empresa MLRForestal?

\section{Revisión de literatura}

La Teca es un árbol de hoja caducada de gran tamaño y copa redondeada cuando crece en condiciones favorables, dando un fuste cilíndrico y limpio que puede alcanzar hasta $30 \mathrm{~m}$ de altura y $80 \mathrm{~cm}$ de diámetro, de rápido crecimiento y con una madera muy apreciada a nivel mundial para la fabricación de muebles (ClubEnsayos, 2012).

Para definir la forma del fuste normalmente se hace mediante el cociente del volumen real y el volumen del cilindro de referencia (producto del área basal y la altura). El factor individual de forma varía con las dimensiones del fuste, con la especie y también difiere de árbol a árbol (Armijos, 2013, pág. 17).

Según el documento técnico del INAFOR "estandarización de unidades de medidas y cálculo de volúmenes de madera", se define como la cantidad de madera estimada en m3 a partir del tocón hasta el ápice del árbol. El volumen puede ser total o comercial, sin incluir las ramas. Depende a partir de que se tomen las alturas, si es altura comercial, o altura total. En latifoliadas normalmente se calcula el volumen comercial del fuste (Instituto Nacional Forestal, 2004).

La tabla de volúmenes Armijos (2013) las define como:

Una expresión tabulada que establece los volúmenes de árboles de acuerdo a una o más de sus dimensiones fáciles de medir, tales como el diámetro normal, la altura y la forma. Algunas de las ventajas de las tablas volumétricas es que son sistemas simples de aplicar y relativamente precisas (pág. 19).

Para México existe el antecedente de Martínez en 1937, quien empleo la ecuación logarítmica de Schumacher para elaborar una tabla de volúmenes de tres especies de pinos, este estudio reporta con sencillez y objetividad el procedimiento empleado 
(Vega et al., 2010). Este estudio marco la pauta para el empleo de estos modelos en ese país, que básicamente se realizaron en bosques de pinos.

Muñoz (2000) evaluó una plantación de Eucalyptus camaldulensis Dehnh establecida en 1961, con el objeto de elaborar cuatro tablas de volumen con y sin corteza, de una y doble entrada, para estimar el volumen en pie del arbolado en el Municipio de Morelia, Mich. Se seleccionaron 139 árboles que fueron derribados, troceados y cubicados, mediante la fórmula de Smalian; posteriormente se probaron once modelos de regresión para las tablas de una entrada y ocho para los de doble entrada. (Valdovinos, 2006)

El factor de forma es una característica que tiene cada especie, pero el Instituto Nacional Forestal (INAFOR) ha establecido utilizar un valor de 0.70 para todas las especies latifoliadas y de 0.47 para coníferas a nivel nacional en Nicaragua siendo estos un valor estándar. (Instituto Nacional Forestal, 2004)

Según Ojeda (1982-1983) existen diferencias en cuanto a los valores del factor de forma entre la familia Leguminosae y la familia Lauraceae llegando a un margen de diferencia de 0.183 ósea un $18.3 \%$, lo que confirma que es importante en el proceso de datos usar factores de forma propios para cada familia y si fuera posible llegar a factores para cada especie. En el caso de las familias Sapotaceae y Lauraceae llega a una diferencia de $13.4 \%$.

En Bolivia el factor de forma que se utiliza para las especies latifoliadas como lo es teca es de 0,65 así lo destaca Heinsdijk en su documento titulado Propuesta para la elaboración de tablas volumétricas y/o factores de forma 1997, dato que coincide relativamente con la investigación realizada en Ecuador por Armijos Guzmán Darwin David con un promedio general de 0.64. (Armijos, 2013).

\section{Materiales y métodos}

La presente investigación se realizó en plantaciones forestales de Tectona grandis L.F 2011 ubicadas en la comunidad de Waspado municipio de Siuna, empresa MLRForestal, el tipo de estudio es predictivo, transversal y prospectivo porque se hicieron estimaciones de índices y modelos matemáticos para la estimación de volumen, la unidad de análisis son los árboles seleccionados para hacer el levantamiento en campo, el marco muestral fueron las tres fincas en las que se realizó el levantamiento de datos en campo (Danli con un total de 58.87 ha, Waspado con 86.28 ha y Mutiwas con 52.85 ha) para un total de 198 ha.

La muestra equivale a 255 árboles en 51 parcelas distribuidos sistemáticamente a una distancia de $100 \mathrm{~m}$ en donde cada parcela contiene 5 árboles entre ellos dominantes, Codominantes y suprimidos, dentro de cada parcela los árboles se encontraban a una 
distancia aproximada de 6 a $8 \mathrm{~m}$. Las variables medidas en campo fueron: diámetro del tocón, diámetro con corteza, diámetro sin corteza, longitud del tocón, longitud de las trozas, longitud de la punta, y altura total, pues son las que se necesitaron para realizar los cálculos al momento de procesar la información.

Los datos levantados en campo se digitalizaron en Excel, posteriormente fueron procesados y analizados mediante diferentes fórmulas para obtener el volumen real y así estimar el Factor de Forma para Tectona grandis L.F.

Con los volúmenes reales se procedió a encontrar un modelos matemático, que más se ajustara a los datos y posteriormente generar una tabla de volúmenes de doble entrada que permitiera hacer estimaciones de volúmenes.

Mediante comparación algorítmica de valores encontrados en las plantaciones se pudo determinar numéricamente las varianzas en los volúmenes provenientes de factores de forma nacionales, el factor de forma encontrado y modelos matemáticos. Para esto se hizo una tabla comparativa para encontrar las diferencias volumétricas.

\section{Resultados y discusión}

\section{Cuantificación del volumen real de Tectona grandis L.F.}

El volumen real de los árboles fue calculado por parte: para el tocón se utilizó la fórmula del cilindro, seguidamente se calculó el volumen cada dos metros utilizando la fórmula de Newton; en el caso que al final no se tuviese trozas de dos metros, se utilizó la fórmula de Smalian para calcular el volumen de esta troza y por último se determinó el volumen de la punta, utilizando la fórmula del paraboloide. Para obtener el volumen total del árbol se sumaron todos los volúmenes anteriores, este procedimiento se repitió para cada uno de los árboles de la muestra. Una vez determinado el volumen real de cada árbol, se procedió a determinar la sumatoria, factor de forma promedio, desviación estándar, coeficiente de variación, Error estándar, límite superior e inferior y error relativo; con una confiabilidad del $95 \%$.

Tabla 1: Estimación del volumen real y factor de forma para un árbol.

\begin{tabular}{|l|l|l|l|c|}
\hline $\begin{array}{c}\text { Altura de la } \\
\text { Sección } \mathbf{( m )}\end{array}$ & $\begin{array}{c}\text { Diámetro } \\
\mathbf{( c m )}\end{array}$ & $\begin{array}{c}\text { Área de la sección } \\
\left(\mathbf{S} \text { en } \mathbf{~ m}^{\mathbf{2}}\right)\end{array}$ & Volumen $\mathbf{( m \mathbf { 3 } )}$ & $\begin{array}{c}\text { Fórmula } \\
\text { Cilindro } \\
V c=S_{0} h_{0}\end{array}$ \\
\hline
\end{tabular}




\begin{tabular}{|c|c|c|c|c|}
\hline $\begin{array}{l}\text { Altura de la } \\
\text { Sección (m) }\end{array}$ & $\begin{array}{l}\text { Diámetro } \\
(\mathrm{cm})\end{array}$ & $\begin{array}{l}\text { Área de la sección } \\
\left.\text { (S en } \mathrm{m}^{2}\right)\end{array}$ & Volumen (m3) & Fórmula \\
\hline 1.30 & 11.7 & 0.01075132 & & \multirow{8}{*}{$\begin{array}{c}\text { Newton } \\
v_{n}=\frac{L}{6}\left(S_{0}+4 S_{m}+S_{1}\right)\end{array}$} \\
\hline 2.30 & 11.3 & 0.01002875 & 0.02325852 & \\
\hline 3.30 & 9.8 & 0.00754296 & & \\
\hline 4.30 & 9.3 & 0.00679291 & 0.0156645 & \\
\hline 5.30 & 8.1 & 0.005153 & & \\
\hline 6.30 & 5.1 & 0.00204282 & 0.00981591 & \\
\hline 7.30 & 4.2 & 0.00138544 & & \\
\hline 8.30 & 3.3 & 0.0008553 & 0.0028133 & \\
\hline 9.30 & 2.1 & 0.00034636 & 0.00060083 & $\begin{array}{c}\text { Smalian } \\
V_{S}=\left(\frac{S_{0}+S_{1}}{2}\right) * L\end{array}$ \\
\hline Longitud de punta & 0.28 & & $6.4654 \mathrm{E}-05$ & $\begin{array}{c}\text { Paraboloide } \\
V_{p a}=\frac{S_{0} h_{0}}{2}\end{array}$ \\
\hline Diámetro de base & 15.7 & Volumen total (m3) & 0.0572 & \\
\hline \multicolumn{3}{|c|}{ Volumen del cilindro (m3) } & 0.1030 & \\
\hline Factor de forma & & & 0.56 & \\
\hline
\end{tabular}

Tabla 2: Tablas Resultados de los parámetros estadísticos por cada una de las fincas.

\begin{tabular}{|l|l|l|l|}
\hline \multirow{2}{*}{ Parámetros Estadísticos } & \multicolumn{3}{c|}{ Fincas } \\
\cline { 2 - 4 } & \multicolumn{1}{|c|}{ Waspado } & \multicolumn{1}{c|}{ Mutiwas } & \multicolumn{1}{c|}{ Danli } \\
\hline Número de árboles & 30 & 125 & 100 \\
\hline Sumatoria & 16.24 & 70.98 & 53.26 \\
\hline Factor forma Promedio & 0.54 & 0.57 & 0.53 \\
\hline Desviación Estándar & 0.05 & 0.07 & 0.08 \\
\hline Coeficiente de Variación & 9.04 & 12.78 & 15.77 \\
\hline Error Estándar & 0.01 & 0.01 & 0.01 \\
\hline límite superior & 0.56 & 0.58 & 0.55 \\
\hline límite inferior & 0.52 & 0.56 & 0.52 \\
\hline Error Relativo (\%) & 3.38 & 2.24 & 3.12 \\
\hline
\end{tabular}

En la finca Waspado se encontró un factor de forma promedio de o.54, una desviación estándar de 0.05 indicando que la variación de los datos es mínima; el coeficiente 
de variación con respeto a la media es de 9.04, indicando que la estimación es precisa, los limites superior 0.56 he inferior 0.52 con un error relativo de $3.38 \%$.

En la finca Mutiwas presenta el factor de forma promedio más alto 0.57 , la desviación estándar 0.07 lo cual nos indica que hay mayor variación en las muestras, el coeficiente de variación 12.78 también es alto, con un error relativo de 2.24.

La finca de Mutiwas tiene diferencia significativa con respecto a las demás fincas el factor de forma está por encima de los límites de confianza de la finca Danlí y Waspado, pues los árboles son más cilíndricos ya que el tratamiento silvicultural que se le ha dado ha sido constante.

La finca Danlí presenta un factor de forma de 0.53, la desviación estándar que se presenta es el mayor 0.08 lo cual indica que la plantación es irregular dando así un 15.77 de coeficiente de variación, con un error relativo de 3.12.

El factor de forma general encontrado es de o.55 en comparación con (David A $G$, 2013) investigación realizada en Ecuador donde el factor de forma para la teca fue de 0.55 .

Según (Castedo, 2011, pág. 47) en una investigación realizada en Turrialba Costa Rica, el factor de forma con corteza encontrado para la Gmelina arbórea es de $0.51 \mathrm{el}$ cual difiere del encontrado que es de 0.55 . Indicando que el factor de forma de forma varía de acuerdo a la especie.

\section{Modelo matemático para las estimaciones de volumen reales de la Tectona grandis L.F.}

Se probaron los modelos matemáticos para ver cual se ajustaba mejor a los datos determinando un modelo potencial de variable combinada, la nube de puntos que muestra la gráfica tiene correlación positiva alta. A continuación, se presenta la gráfica de nubes de punto donde se muestra el modelo matemático que fue seleccionado y que fue aplicado en el municipio de Siuna - RACCN. 


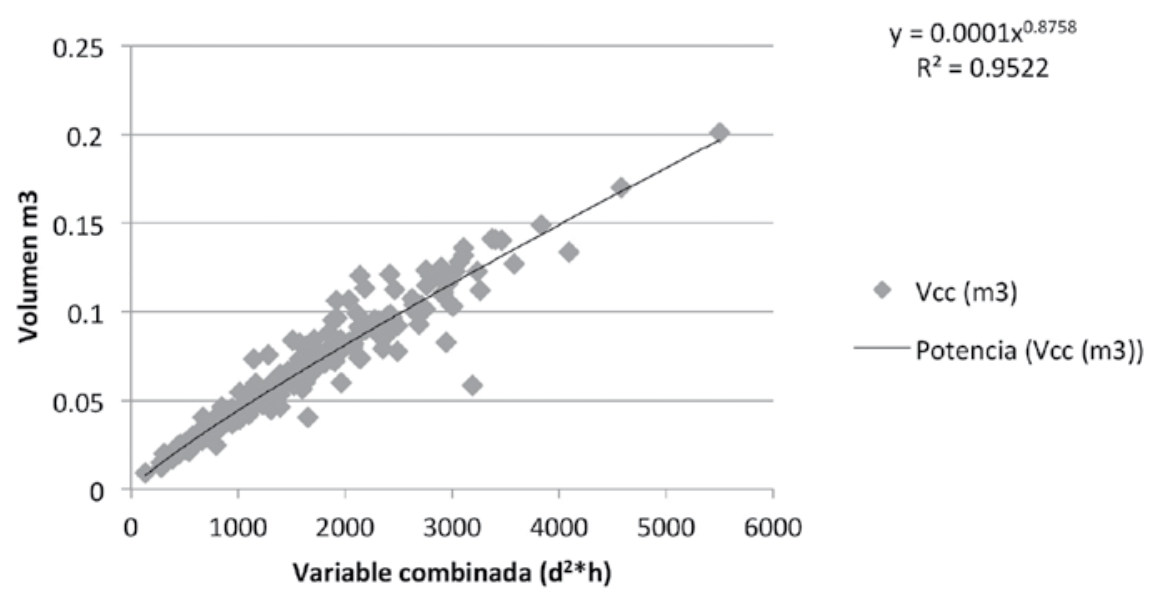

Figura 1: Diagrama de dispersión por nube de puntos.

El modelo utilizado $V=0.0001\left(\mathrm{~d}^{2 *} \mathrm{~h}\right) 0.8758 \mathrm{R}^{2}=0.9522$ corresponde a un modelo potencial.

$\mathrm{R}^{2}=$ Coeficiente de determinación que nos indica que tan ajustados o dispersos están los datos con respecto a la línea de tendencia.

$$
V=\log \alpha+\beta \log \left(d^{2} h\right)
$$

Dónde: $V=$ Volumen, $\alpha=$ intersección al eje $\mathrm{x}, \beta=$ Pendiente, $\mathrm{d}=$ Diámetro $(\mathrm{cm})$, $\mathrm{h}=\operatorname{Altura}(\mathrm{m})$

Con el modelo matemático se procedió a elaborar una tabla de volúmenes que se muestra a continuación en la tabla 3. 


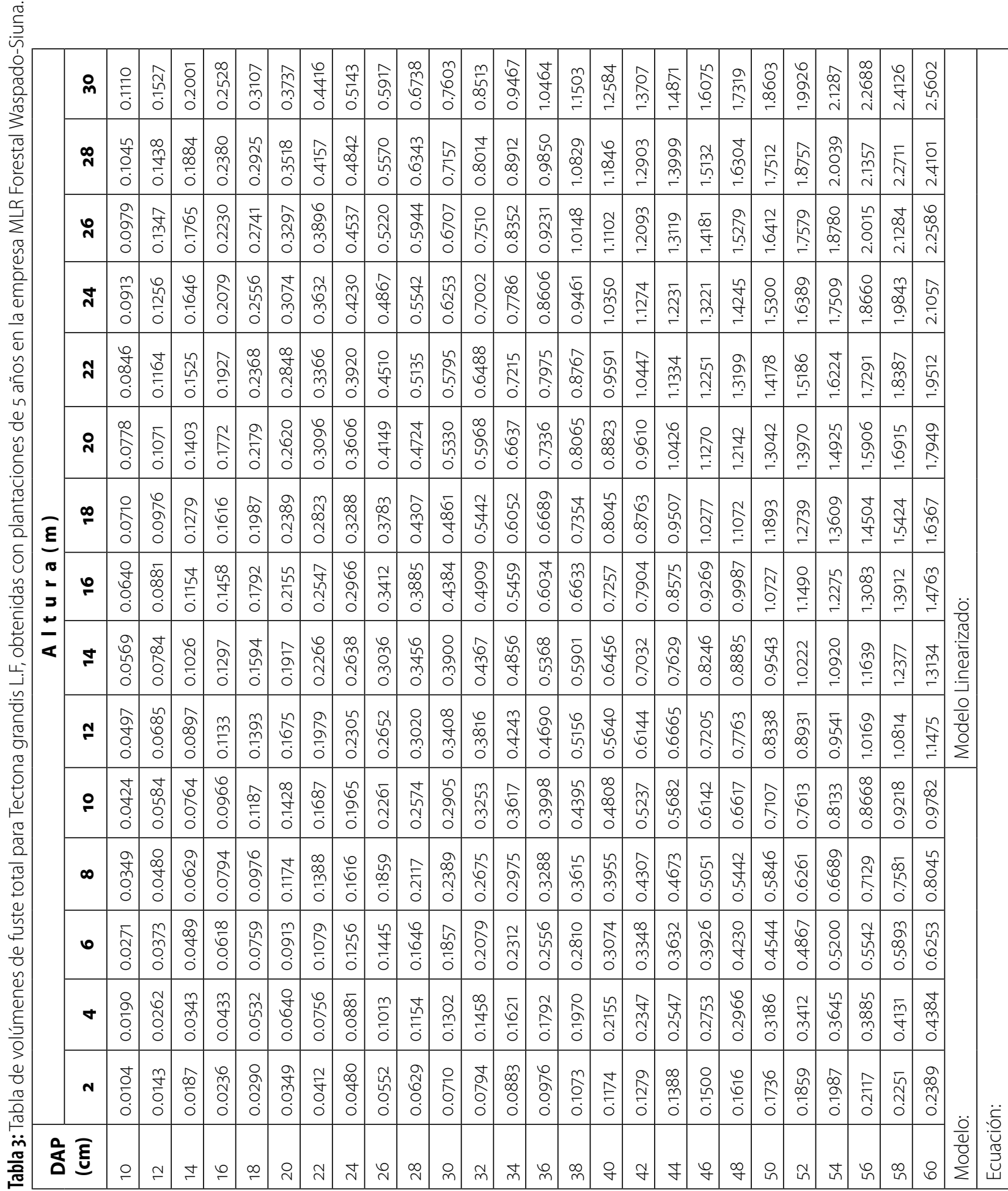




\section{Diferencias volumétricas entre el factor de forma calculado, modelo matemático y factor de forma nacional para las especies latifoliadas}

En la tabla 4., podemos observar las diferencias volumétricas y porcentuales entre el volumen real y factor de forma calculado, modelo matemático y factor de forma nacional, obteniendo menor error el factor de forma calculado y el modelo matemático, lo cual nos indica que el cálculo de volumen con estos dos es más preciso, mientras que al calcular el volumen con el factor de forma nacional el error cometido es de 19.83\%.

Tabla 4: Diferencias volumétricas y porcentuales entre Volumen Real y Volumen con factor de Forma calculado, Modelo Matemático y Factor de Forma Nacional

\begin{tabular}{|c|c|c|c|c|c|c|}
\hline PROMEDIO FINAL & $\begin{array}{l}\text { Dn promedio } \\
(\mathrm{cm})\end{array}$ & $\begin{array}{c}\text { H } \\
\text { promedio } \\
\text { (m) }\end{array}$ & $\begin{array}{l}\text { Volumen real } \\
\text { promedio }\left(\mathrm{m}^{3}\right)\end{array}$ & $\begin{array}{l}\text { Volumen factor de } \\
\text { forma calculado }\left(\mathrm{m}^{3}\right)\end{array}$ & $\begin{array}{l}\text { Volumen con } \\
\text { el modelo } \\
\text { matemático } \\
\left(\mathrm{m}^{3}\right)\end{array}$ & $\begin{array}{l}\text { Volumen con el } \\
\text { factor de forma } \\
\text { Nacional }\left(m^{3}\right)\end{array}$ \\
\hline Vol. $\mathrm{m}^{3}$ & \multirow{3}{*}{11.77} & \multirow{3}{*}{10.05} & \multirow{3}{*}{0.0639} & 0.060161495 & 0.056675714 & 0.076569175 \\
\hline $\begin{array}{l}\text { Diferencia Vol. Real } \\
\text { m3 }\end{array}$ & & & & -0.00373411 & -0.00721989 & 0.012673573 \\
\hline Porcentaje de Error & & & & -5.84 & -11.30 & 19.83 \\
\hline
\end{tabular}

Forma calculado, Modelo Matemático y Factor de Forma Nacional.

Estos datos coinciden con Ojeda (1983) cuando plantea que el factor de forma se debe de calcular familias y si es posible por especies para disminuir el porcentaje de error en el cálculo de volúmenes.

\section{Conclusiones}

En la determinación del factor de forma para la Tectona grandis L.F en la empresa MLR-Forestal llegamos a las siguientes conclusiones

$1 \mathrm{El}$ factor de forma general encontrado para la Tectona grandis L.F es de $0.55 \mathrm{el}$ cual difiere del factor de forma nacional, establecido por el Instituto Nacional Forestal (INAFOR) el cual es de 0.70.

2 El modelo matemático propuesto para la construcción de tabla de volúmenes de doble entrada es: $V=0.0001\left(d^{2} * h\right) 0.8758 R^{2}=0.9522$.

3 El porcentaje de error con el factor de forma nacional es de (19.83\%) en comparación con el factor de forma encontrado $(-5.84 \%)$ y el modelo matemático $(-11.30 \%)$. 


\section{Recomendaciones}

A cualquier persona natural o jurídica que se dedique al manejo y aprovechamiento forestal de cualquier especie, instarla a determinar factores de forma para la especie que trabajan, para disminuir el porcentaje de error al momento de hacer estimaciones de volumen.

Al Instituto Nacional Forestal, que promueva las investigaciones relacionadas al crecimiento y desarrollos de los bosques para garantizar un manejo sostenible de los recursos naturales.

A la universidad, que promueva la investigación forestal con fines de garantizar la sostenibilidad de los bosques.

\section{Lista de referencias}

Castedo, P. A. (2011). Factor de forma para la Gmelina arborea. Riomba-Ecuador.

David, A. G. (2013). Construccion de tablas volumetricas y calculo del factor de forma (ff) para dos especies, Teca (Tectona grandis) y melina (Gmelina arborea). Riobamba, Ecuador.

David, A. G. (2013). Construccion de tablas volumetricas y calculo del factor de forma (ff) para la especie Teca ( Tectona grandis) y melina(gmelina arborea). Ecuador.

Instituto Nacional Forestal. (2004). Estandarizacion de unidades de medidas y calculo de volumenes de madera. tecnico, INAFOR, Managua, Managua. Recuperado el febrero de 2017

Ojeda, W. (1983). Factor de forma preliminar para seis familias de especies forestales tropicales. Perú.

Valdovinos, J. R. (2006). Sistema de cubicación para Eucalyptus grandis y E. urophylla en los límites de Veracruz y Oaxaca. Texcoco, Mexico.

Vega, C. F., \& Maldonado., H. R. (2010). Dendrometria. Mexico: Chapingo.

Zaragoza. (2009). El cultivo de la TECA. 